\title{
INTEGRALLY CLOSED IDEALS ON LOG TERMINAL SURFACES ARE MULTIPLIER IDEALS
}

\author{
KeVIN TUCKer
}

Abstract. We show that all integrally closed ideals on log terminal surfaces are multiplier ideals by extending an existing proof for smooth surfaces.

\section{Introduction}

Consider a scheme $X=\operatorname{Spec} \mathcal{O}_{X}$, where $\mathcal{O}_{X}$ is a two-dimensional local normal domain essentially of finite type over $\mathbb{C}$. Our purpose is to partially address the following question, raised in [6]

Question. If $X$ has a rational singularity, is every integrally closed ideal which is contained in $\mathcal{J}\left(X, \mathcal{O}_{X}\right)$ a multiplier ideal?

Here, $\mathcal{J}\left(X, \mathfrak{a}^{\lambda}\right)$ denotes the multiplier ideal corresponding to an ideal $\mathfrak{a} \subseteq \mathcal{O}_{X}$ with coefficient $\lambda \in \mathbb{Q}_{>0}$. When $X$ is regular, an affirmative answer was given concurrently by 8 and $[3$. Our main result is to generalize their methods to prove the following:

Theorem 1.1. Suppose $X$ has log terminal singularities. Then every integrally closed ideal is a multiplier ideal.

Log terminal singularities satisfy $\mathcal{J}\left(X, \mathcal{O}_{X}\right)=\mathcal{O}_{X}$ by definition, and are necessarily rational (see Theorem 5.22 in [4]). Thus, Theorem 1.1 gives a complete answer to the above question in this case.

There are several difficulties in trying to extend the techniques used in 8 . One must show that successful choices can be made in the construction (specifically, the choice of $\epsilon$ and $N$ in Lemma 2.2 of 8 ). Here, it is essential that $X$ has log terminal singularities. Further problems arise from the failure of unique factorization to hold for integrally closed ideals. As $X$ is not necessarily factorial, we may no longer reduce to the finite colength case. In addition, the crucial contradiction argument which concludes the proof in [8] does not apply. These nontrivial difficulties are overcome by using a relative numerical decomposition for divisors on a resolution over $X$. Further, appropriately interpreted, the proof of Theorem 1.1 applies over an algebraically closed field of arbitrary characteristic.

Our presentation is self-contained and elementary. Section 2 contains background material covering the relative numerical decomposition, antinef closures, and some computations using generic sequences of blowups. Section 3 is dedicated to the constructions and arguments in the proof of Theorem 1.1

Received by the editors September 17, 2008.

The author was partially supported by the NSF under grant DMS-0502170. 


\section{Background}

2.1. Relative Numerical Decomposition. For the remainder, we will consider a scheme $X=\operatorname{Spec} \mathcal{O}_{X}$, where $\mathcal{O}_{X}$ is a two-dimensional local normal domain essentially of finite type over an algebraically closed field of arbitrary characteristic. Let $x \in X$ be the unique closed point, and suppose $f: Y \rightarrow X$ is a projective birational morphism such that $Y$ is regular and $f^{-1}(x)$ is a simple normal crossing divisor. Let $E_{1}, \ldots, E_{u}$ be the irreducible components of $f^{-1}(x)$, and $\Lambda=\oplus_{i} \mathbb{Z} E_{i} \subset \operatorname{Div}(Y)$ the lattice they generate.

The intersection pairing $\operatorname{Div}(Y) \times \Lambda \rightarrow \mathbb{Z}$ induces a negative definite $\mathbb{Q}$-bilinear form on $\Lambda_{\mathbb{Q}}$ (see [1] for an elementary proof). Consequently, there is a dual basis $\check{E}_{1}, \ldots, \check{E}_{u}$ for $\Lambda_{\mathbb{Q}}$ defined by the property that

$$
\check{E}_{i} \cdot E_{j}=-\delta_{i j}=\left\{\begin{array}{cc}
-1 & i=j \\
0 & i \neq j
\end{array} .\right.
$$

Recall that a divisor $D \in \operatorname{Div}_{\mathbb{Q}}(Y)$ is said to be $f$-antinef if $D \cdot E_{i} \leq 0$ for all $i=1, \ldots, u$. In this case, $D$ is effective if and only if $f_{*} D$ is effective (see Lemma 3.39 in [4]). In particular, $\check{E}_{1}, \ldots, \check{E}_{u}$ are effective.

If $C \in \operatorname{Div}_{\mathbb{Q}}(X)$, we define the numerical pullback of $C$ to be the unique $\mathbb{Q}$-divisor $f^{*} C$ on $Y$ such that $f_{*} f^{*} C=C$ and $f^{*} C \cdot E_{i}=0$ for all $i=1, \ldots, u$. Note that, when $C$ is Cartier or even $\mathbb{Q}$-Cartier, this agrees with the standard pullback of $C$. If $D \in \operatorname{Div}_{\mathbb{Q}}(Y)$, we have

$$
D=f^{*} f_{*} D+\sum_{i}\left(-D \cdot E_{i}\right) \check{E}_{i} .
$$

We shall refer to this as a relative numerical decomposition for $D$. Note that, even when $D$ is integral, both $f^{*} f_{*} D$ and $\check{E}_{1}, \ldots, \check{E}_{u}$ are likely non-integral. The fact that $f^{*} f_{*} D$ and $\check{E}_{1}, \ldots, \check{E}_{u}$ are always integral divisors when $X$ is smooth and $D$ is integral is equivalent to the unique factorization of integrally closed ideals. See [7] for further discussion.

2.2. Antinef Closures and Global Sections. Suppose now that $D^{\prime}=\sum_{E} a_{E}^{\prime} E$ and $D^{\prime \prime}=\sum_{E} a_{E}^{\prime \prime} E$ are $f$-antinef divisors, where the sums range over the prime divisors $E$ on $Y$. It is easy to check that $D^{\prime} \wedge D^{\prime \prime}=\sum_{E} \min \left\{a_{E}^{\prime}, a_{E}^{\prime \prime}\right\} E$ is also $f$ antinef. Further, any integral $D \in \operatorname{Div}(Y)$ is dominated by some integral $f$-antinef divisor (e.g. $\left(f_{*}^{-1}\right) f_{*} D+M\left(\check{E}_{1}+\cdots+\check{E}_{u}\right)$ for sufficiently large and divisible $\left.M\right)$. In particular, there is a unique smallest integral $f$-antinef divisor $D^{\sim}$, called the $f$ antinef closure of $D$, such that $D^{\sim} \geq D$. One can verify that $f_{*} D=f_{*} D^{\sim}$, and in addition the following important lemma holds (see Lemma 1.2 of [8]). The proof also gives an effective algorithm for computing $f$-antinef closures.

Lemma 2.1. For any $D \in \operatorname{Div}(Y)$, we have $f_{*} \mathcal{O}_{Y}(-D)=f_{*} \mathcal{O}_{Y}\left(-D^{\sim}\right)$.

Proof. Let $s_{D} \in \mathbb{N}$ be the sum of the coefficients of $D^{\sim}-D$ when written in terms of $E_{1}, \ldots, E_{u}$. If $s_{D}=0$, then $D=D^{\sim}$ is $f$-antinef and the statement follows trivially. Else, there is an index $i$ such that $D \cdot E_{i}>0$. As $E_{i} \cdot E_{j} \geq 0$ for $j \neq i$, we must have

$$
D \leq D+E_{i} \leq D^{\sim}=\left(D+E_{i}\right)^{\sim} .
$$


Thus, $s_{D+E_{i}}=s_{D}-1$. By induction, we may assume

$$
f_{*} \mathcal{O}_{Y}\left(-\left(D+E_{i}\right)\right)=f_{*} \mathcal{O}_{Y}\left(-\left(D+E_{i}\right)^{\sim}\right)=f_{*} \mathcal{O}_{Y}\left(-D^{\sim}\right)
$$

and it is enough to show $f_{*} \mathcal{O}_{Y}(-D)=f_{*} \mathcal{O}_{Y}\left(-\left(D+E_{i}\right)\right)$. Consider the exact sequence

$$
0 \longrightarrow \mathcal{O}_{Y}\left(-\left(D+E_{i}\right)\right) \longrightarrow \mathcal{O}_{Y}(-D) \longrightarrow \mathcal{O}_{E_{i}}(-D) \longrightarrow 0 .
$$

Since $\operatorname{deg}\left(\mathcal{O}_{E_{i}}(-D)\right)=-D \cdot E_{i}<0$, we have $f_{*} \mathcal{O}_{E_{i}}(-D)=0$; applying $f_{*}$ yields the desired result.

2.3. Generic Sequences of Blowups. In the proof of Theorem 1.1, we will make use of the following auxiliary construction. Suppose $x^{(i)}$ is a closed point of $E_{i}$ with $x^{(i)} \notin E_{j}$ for $j \neq i$. A generic sequence of $n$-blowups over $x^{(i)}$ is:

$$
Y=Y_{0} \stackrel{\sigma_{1}}{\longleftarrow} Y_{1} \stackrel{\sigma_{2}}{\longleftarrow} \cdots \stackrel{\sigma_{n-1}}{\longleftarrow} Y_{n-1} \stackrel{\sigma_{n}}{\longleftarrow} Y_{n}
$$

where $\sigma_{1}: Y_{1} \rightarrow Y_{0}$ is the blowup of $Y_{0}=Y$ at $x_{1}:=x^{(i)}$, and $\sigma_{k}: Y_{k} \rightarrow Y_{k-1}$ is the blowup of $Y_{k-1}$ at a generic closed point $x_{k}$ of $\left(\sigma_{k-1}\right)^{-1}\left(x_{k-1}\right)$ for $k=2, \ldots, n$. Let $\sigma: Y_{n} \rightarrow Y$ be the composition $\sigma_{n} \circ \cdots \circ \sigma_{1}$. We will denote by $E(1), \ldots, E(u)$ the strict transforms of $E_{1}, \ldots, E_{u}$ on $Y_{n}$. Also, let $E\left(i, x^{(i)}, k\right), k=1, \ldots, n$, be the strict transforms of the $n$ new $\sigma$-exceptional divisors created by the blowups $\sigma_{1}, \ldots, \sigma_{n}$, respectively.

Lemma 2.2. (a.) Let $\sigma: Y_{n} \rightarrow Y$ be a generic sequence of blowups over $x^{(i)} \in E_{i}$. Then one has

$$
\check{E}(i) \leq \check{E}\left(i, x^{(i)}, 1\right) \leq \cdots \leq \check{E}\left(i, x^{(i)}, n\right) .
$$

(b.) Suppose $D \in \operatorname{Div}\left(Y_{n}\right)$ is an integral $(f \circ \sigma)$-antinef divisor such that $E_{i}$ is the unique component of $\sigma_{*} D$ containing $x^{(i)}$. If $\operatorname{ord}_{E(i)} D=a_{0}$ and $\operatorname{ord}_{E\left(i, x^{(i)}, k\right)} D=$ $a_{k}$ for $k=1, \ldots, n$, then

$$
a_{0} \leq a_{1} \leq \cdots \leq a_{n}
$$

Further, $a_{0}<a_{n}$ if and only if

$$
\left(\sum_{k=1}^{n}\left(-D \cdot E\left(i, x^{(i)}, k\right)\right) \check{E}\left(i, x^{(i)}, k\right)\right) \geq \check{E}(i) .
$$

Proof. If $n=1$, we have

$$
\begin{gathered}
\check{E}\left(i, x^{(i)}, 1\right)=\left(\sigma^{*} \check{E}_{i}+E\left(i, x^{(i)}, 1\right)\right) \geq \sigma^{*} \check{E}_{i}=\check{E}(i) \\
D=\sigma^{*} \sigma_{*} D+\left(-D \cdot E\left(i, x^{(i)}, 1\right)\right) \check{E}\left(i, x^{(i)}, 1\right) .
\end{gathered}
$$

The general case of both statments follows easily by induction. 


\section{Main Theorem}

3.1. Log Terminal Singularities and Multiplier Ideals. Once more, suppose $x \in X$ is the unique closed point and $f: Y \rightarrow X$ is a projective birational morphism such that $Y$ is regular and $f^{-1}(x)$ is a simple normal crossing divisor. Let $E_{1}, \ldots, E_{u}$ be the irreducible components of $f^{-1}(x)$, and let $K_{Y}$ be a canonical divisor on $Y$. Then $K_{X}:=f_{*} K_{Y}$ is a canonical divisor on $X$. If we write the relative canonical divisor as

$$
K_{f}:=K_{Y}-f^{*} K_{X}=\sum_{i} b_{i} E_{i}
$$

then $X$ has (numerically) $\log$ terminal singularities if and only if $b_{i}>-1$ for all $i=1, \ldots, u$. In this case, when working over $\mathbb{C}, X$ is automatically $\mathbb{Q}$-factorial (see Proposition 4.11 in [4], as well as [2] for recent developments).

If $\mathfrak{a} \subseteq \mathcal{O}$ is an ideal, recall that $f: Y \rightarrow X$ as above is said to be a log resolution of $\mathfrak{a}$ if $\mathfrak{a} \mathcal{O}_{Y}=\mathcal{O}_{Y}(-G)$ for an effective divisor $G$ such that $\operatorname{Ex}(f) \cup \operatorname{Supp}(G)$ has simple normal crossings. In this case, we can define the multiplier ideal of $(X, \mathfrak{a})$ with coefficient $\lambda \in \mathbb{Q}_{>0}$ as

$$
\mathcal{J}\left(X, \mathfrak{a}^{\lambda}\right)=f_{*} \mathcal{O}_{Y}\left(\left\lceil K_{f}-\lambda G\right\rceil\right) .
$$

See [9] for an introduction in a similar setting, or [5] for a more comprehensive overview. Also recall that $\mathfrak{a}$ is integrally closed if and only if

$$
\mathfrak{a}=f_{*} \mathcal{O}_{Y}(-G) .
$$

3.2. Choosing $\mathfrak{a}$ and $\lambda$. We now begin the proof of Theorem 1.1. For the remainder, assume $X$ is $\log$ terminal, and let $I \subseteq \mathcal{O}_{X}$ be an integrally closed ideal. In this section, we construct another ideal $\mathfrak{a} \subseteq \mathcal{O}_{X}$ along with a coefficient $\lambda \in \mathbb{Q}_{>0}$; and in the following section it will be shown that $\mathcal{J}\left(X, \mathfrak{a}^{\lambda}\right)=I$. Let $f: Y \rightarrow X$ a log resolution of $I$ with exceptional divisors $E_{1}, \ldots, E_{u}$. Suppose $I \mathcal{O}_{Y}=\mathcal{O}_{Y}\left(-F^{0}\right)$, and write

$$
\begin{gathered}
K_{f}=\sum_{i=1}^{u} b_{i} E_{i} \\
F^{0}=\left(f^{-1}\right) f_{*}\left(F^{0}\right)+\sum_{i=1}^{u} a_{i} E_{i} .
\end{gathered}
$$

Choose $0<\epsilon<1 / 2$ such that $\left\lfloor\epsilon\left(f_{*}^{-1}\right) f_{*}\left(F^{0}\right)\right\rfloor=0$ and

$$
\epsilon\left(a_{i}+1\right)<1+b_{i}
$$

for $i=1, \ldots, u$. Note that, since $X$ is $\log$ terminal, $1+b_{i}>0$ and any sufficiently small $\epsilon>0$ will do. Let $n_{i}:=\left\lfloor\frac{1+b_{i}}{\epsilon}-\left(a_{i}+1\right)\right\rfloor \geq 0$, and $e_{i}:=\left(-F^{0} \cdot E_{i}\right)$. Choose $e_{i}$ distinct closed points $x_{1}^{(i)}, \ldots, x_{e_{i}}^{(i)}$ on $E_{i}$ such that $x_{j}^{(i)} \notin \operatorname{Supp}\left(\left(f^{-1}\right) f_{*}\left(F^{0}\right)\right)$ and $x_{j}^{(i)} \notin E_{l}$ for $l \neq i$. Denote by $g: Z \rightarrow Y$ the composition of $n_{i}$ generic blowups at each of the points $x_{j}^{(i)}$ for $j=1, \ldots, e_{i}$ and $i=1, \ldots, u$. As in Section 2.3 , denote by $E(1), \ldots, E(u)$ the strict transforms of $E_{1}, \ldots, E_{u}$, and $E\left(i, x_{j}^{(i)}, 1\right), \ldots, E\left(i, x_{j}^{(i)}, n_{i}\right)$ the strict transforms of the $n_{i}$ exceptional divisors over $x_{j}^{(i)}$. 
Let $h:=f \circ g, F=g^{*}\left(F^{0}\right)$, and choose an effective $h$-exceptional integral divisor $A$ on $Z$ such that $-A$ is $h$-ample. It is easy to see that

$$
K_{g}=\sum_{i=1}^{u} \sum_{j=1}^{e_{i}} \sum_{k=1}^{n_{i}} k E\left(i, x_{j}^{(i)}, k\right)
$$

and one checks

$$
K_{g} \cdot E(i)=e_{i} \quad K_{g} \cdot E\left(i, x_{j}^{(i)}, k\right)=\left\{\begin{array}{cc}
0 & k \neq n_{i} \\
-1 & k=n_{i}
\end{array} .\right.
$$

It follows immediately that $F+K_{g}$ is $h$-antinef. Choose $\mu>0$ sufficiently small that

$$
\left\lfloor(1+\epsilon)\left(F+K_{g}+\mu A\right)-K_{h}\right\rfloor=\left\lfloor(1+\epsilon)\left(F+K_{g}\right)-K_{h}\right\rfloor .
$$

As $-\left(F+K_{g}+\mu A\right)$ is $h$-ample, there exists $N>>0$ such that $G:=N\left(F+K_{g}+\mu A\right)$ is integral and $-G$ is relatively globally generated ${ }^{1}$ In other words, $\mathfrak{a}:=h_{*} \mathcal{O}_{Z}(-G)$ is an integrally closed ideal such that $\mathfrak{a} \mathcal{O}_{Z}=\mathcal{O}_{Z}(-G)$. Set $\lambda=\frac{1+\epsilon}{N}$.

3.3. Conclusion of Proof. Here, we will show $\mathcal{J}\left(X, \mathfrak{a}^{\lambda}\right)=I=h_{*} \mathcal{O}_{Z}(-F)$. Since

$$
\mathcal{J}\left(X, \mathfrak{a}^{\lambda}\right)=h_{*} \mathcal{O}_{Z}\left(\left\lceil K_{h}-\lambda G\right\rceil\right)=h_{*} \mathcal{O}_{Z}\left(-\left\lfloor\lambda G-K_{h}\right\rfloor\right),
$$

by Lemma 2.1, it suffices to show $F^{\prime}:=\left\lfloor\lambda G-K_{h}\right\rfloor^{\sim}=F$. In particular, we have reduced to showing a purely numerical statement.

Lemma 3.1. We have $F^{\prime} \leq F$ and $h_{*} F^{\prime}=h_{*} F$. In addition, for $i=1, \ldots, u$ and $j=1, \ldots, e_{i}$,

$$
\operatorname{ord}_{E\left(i, x_{j}^{(i)}, n_{i}\right)}\left(F^{\prime}\right)=\operatorname{ord}_{E\left(i, x_{j}^{(i)}, n_{i}\right)}(F)=\operatorname{ord}_{E(i)}(F) .
$$

Proof. Since $F^{\prime}=\left\lfloor\lambda G-K_{h}\right\rfloor^{\sim}$ and $F$ is $h$-antinef $(-F$ is relatively globally generated), it suffices to show these statements with $\left\lfloor\lambda G-K_{h}\right\rfloor$ in place of $F^{\prime}$. By (2), we have

$$
\begin{aligned}
\left\lfloor\lambda G-K_{h}\right\rfloor & =\left\lfloor(1+\epsilon)\left(F+K_{g}\right)-K_{h}\right\rfloor \\
& =F+\left\lfloor\epsilon\left(F+K_{g}\right)-g^{*} K_{f}\right\rfloor .
\end{aligned}
$$

Since $\left\lfloor\epsilon\left(f_{*}^{-1}\right) f_{*} F^{0}\right\rfloor=0$, it follows immediately that $h_{*}\left\lfloor\lambda G-K_{h}\right\rfloor=h_{*} F$. For the remaining two statements, consider the coefficients of $\epsilon\left(F+K_{g}\right)-g^{*} K_{f}$. Along $E(i)$, we have $\epsilon a_{i}-b_{i}$, which is less than one by choice of $\epsilon$. Along $E\left(i, x_{j}^{(i)}, k\right)$, we have $\epsilon\left(a_{i}+k\right)-b_{i}$. This expression is greatest when $k=n_{i}$, where our choice of $n_{i}$ guarantees

$$
0 \leq \epsilon\left(a_{i}+n_{i}\right)-b_{i}<1 .
$$

It follows that $\left\lfloor\lambda G-K_{h}\right\rfloor \leq F$, with equality along $E\left(i, x_{j}^{(i)}, n_{i}\right)$.

Lemma 3.2. For each $i=1, \ldots, u$,

$$
\left(-F^{\prime} \cdot E(i)\right) \check{E}(i)+\sum_{j=1}^{e_{i}} \sum_{k=1}^{n_{i}}\left(-F^{\prime} \cdot E\left(i, x_{j}^{(i)}, k\right)\right) \check{E}\left(i, x_{j}^{(i)}, k\right) \geq \quad(-F \cdot E(i)) \check{E}(i) .
$$

\footnotetext{
${ }^{1}$ Over $\mathbb{C}$, as $X$ is $\log$ terminal, it also has rational singularities and by Theorem 12.1 of 7 it follows that $-\left(F+K_{g}\right)$ is already globally generated without the addition of $-A$. However, the above approach seems more elementary, and avoids unnecessary reference to these nontrivial results.
} 
Proof. If $\operatorname{ord}_{E(i)} F^{\prime}=\operatorname{ord}_{E(i)} F$, as $F^{\prime} \leq F$ we have $F^{\prime} \cdot E(i) \leq F \cdot E(i)$ and the conclusion follows as $\check{E}(i)$ and $\check{E}\left(i, x_{j}^{(i)}, k\right)$ are effective and $F^{\prime}$ is $h$-antinef. Otherwise, if $\operatorname{ord}_{E(i)} F^{\prime}<\operatorname{ord}_{E(i)} F=\operatorname{ord}_{E\left(i, x_{j}^{(i)}, n_{i}\right)} F^{\prime}$, then for each $j=1, \ldots, e_{i}$ we saw in Lemma 2.2 b that

$$
\sum_{k=1}^{n_{i}}\left(-F^{\prime} \cdot E\left(i, x_{j}^{(i)}, k\right)\right) \check{E}\left(i, x_{j}^{(i)}, k\right) \geq \check{E}(i) .
$$

Summing over all $j$ gives the desired conclusion.

We now finish the proof by showing that $F^{\prime} \geq F$. Using the relative numerical decomposition (1) and the previous two Lemmas, we compute

$$
\begin{aligned}
F^{\prime} & =h^{*} h_{*} F^{\prime}+\sum_{i=1}^{u}\left(-F^{\prime} \cdot E(i)\right) \check{E}(i)+\sum_{i=1}^{u} \sum_{j=1}^{e_{i}} \sum_{k=1}^{n_{i}}\left(-F^{\prime} \cdot E\left(i, x_{j}^{(i)}, k\right)\right) \check{E}\left(i, x_{j}^{(i)}, k\right) \\
& =h^{*}\left(h_{*} F\right)+\sum_{i=1}^{u}\left(\left(-F^{\prime} \cdot E(i)\right) \check{E}(i)+\sum_{j=1}^{e_{i}} \sum_{k=1}^{n_{i}}\left(-F^{\prime} \cdot E\left(i, x_{j}^{(i)}, k\right)\right) \check{E}\left(i, x_{j}^{(i)}, k\right)\right) \\
& \geq h^{*} h_{*} F+\sum_{i=1}^{u}(-F \cdot E(i)) \check{E}(i)=F .
\end{aligned}
$$

This concludes the proof of Theorem 1.1

\section{References}

[1] M. Artin, On isolated rational singularities of surfaces, Amer. J. Math. 88 (1966) 129-136.

[2] T. de Fernex and C. Hacon, Singularities on Normal Varieties, Compos. Math. 145 (2009) 393-414.

[3] C. Favre and M. Jonsson, Valuations and multiplier ideals, J. Amer. Math. Soc. 18 (2005), no. 3, 655-684 (electronic).

[4] J. Kollár and S. Mori, Birational geometry of algebraic varieties, Vol. 134 of Cambridge Tracts in Mathematics, Cambridge University Press, Cambridge (1998), ISBN 0-521-63277-3. With the collaboration of C. H. Clemens and A. Corti, Translated from the 1998 Japanese original.

[5] R. Lazarsfeld, Positivity in algebraic geometry. II, Vol. 49 of Ergebnisse der Mathematik und ihrer Grenzgebiete. 3. Folge. A Series of Modern Surveys in Mathematics [Results in Mathematics and Related Areas. 3rd Series. A Series of Modern Surveys in Mathematics], Springer-Verlag, Berlin (2004), ISBN 3-540-22534-X. Positivity for vector bundles, and multiplier ideals.

[6] R. Lazarsfeld, K. Lee, and K. E. Smith, Syzygies of multiplier ideals on singular varieties, Michigan Math. J. 57 (2008) 511-521. Special volume in honor of Melvin Hochster.

[7] J. Lipman, Rational singularities, with applications to algebraic surfaces and unique factorization, Inst. Hautes Études Sci. Publ. Math. (1969), no. 36, 195-279.

[8] J. Lipman and K.-i. Watanabe, Integrally closed ideals in two-dimensional regular local rings are multiplier ideals, Math. Res. Lett. 10 (2003), no. 4, 423-434.

[9] K. Tucker, Jumping Numbers on Algebraic Surfaces with Rational Singularities (2009). To appear in Transactions of the AMS. arXiv:0801.0734v2.

Department of Mathematics, University of Michigan, 2704 East Hall, 525 East UniverSity Avenue, Ann Arbor, MI 48109-1109

E-mail address: kevtuck@umich.edu 